\title{
Sperm donation and its application in China: a 7-year multicenter retrospective study
}

\author{
Ping Ping ${ }^{1, *}$, Wen-Bing Zhu ${ }^{2, *}$, Xin-Zong Zhang ${ }^{3}$, Yu-Shan Li ${ }^{4}$, Quan-Xian Wang ${ }^{4}$, Xiao-Rong Cao ${ }^{1}$, Yong Liu ${ }^{1}$, \\ Hui-Li Dai ${ }^{1}$, Yi-Ran Huang ${ }^{1}$ and Zheng $\mathrm{Li}^{1}$
}

Sperm donation in China is different from that in other countries due to cultural, social and political factors. This research presents the current status of sperm donation in Mainland China and highlights some problems. Between January 2003 and December 2009, 19471 sperm donors were screened totally and 6467 donors (33.2\%) were recruited. The primary reasons for non-recruitment were either inadequate semen parameters $(55.0 \%)$ or positive results for sexually transmitted diseases $(7.9 \%)$. There were $327(1.7 \%)$ qualified donors who withdrew from the program because of frustration related to failed semen parameters, participation merely for free medical tests or job transfer. A questionnaire investigating donor intention, as well as other concerns associated with sperm donation, was distributed to 516 potential donors. All potential donors indicated their primary motivation as altruism, while $90.9 \%$ mentioned monetary reward as a second motivating factor. Approximately $93.4 \%$ of donors expressed some apprehension about the risk of consanguineous mating and the protection of their identity. Over the past 7 years, 488389 vials of donors' semen have been cryopreserved. In 36438 artificial insemination with donor sperm (AID) cycles, the clinical pregnancy rate was $23.9 \%$ and the live birth rate was $16.6 \%$. In 7148 in vitro fertilization cycles, the clinical pregnancy rate was $45.8 \%$ and the live birth rate was $35.2 \%$. Human sperm banks have been strictly monitored to ensure that each sperm donor can only impregnate five women nationwide. There is still a large gap between the supply and demand for sperm donation which may be solved by updated guidelines.

Asian Journal of Andrology (2011) 13, 644-648; doi:10.1038/aja.2011.20; published online 30 May 2011

Keywords: artificial insemination with donor sperm; donor; in vitro fertilization; sperm donation

\section{INTRODUCTION}

The first human pregnancy using frozen sperm was reported in 1953. Due to the moral and legal controversies surrounding the use of artificial insemination, however, the first real breakthroughs in human sperm banking did not occur until 10 years later at the Eleventh International Congress of Genetics. ${ }^{1}$ Over the last 30 years, cryostorage techniques have been markedly improved and long-term storage in liquid nitrogen is now a standard and well-established procedure. Therapeutic insemination using donor sperm has been established as a highly effective and well-accepted means of achieving conception for couples with severe issues of male infertility. Since 1993, intracytoplamic sperm injection (ICSI) has become widely available, enabling males with obstructive azoospermia and severe oligospermia to have genetically related offspring. ${ }^{1}$ Microdissection testicular sperm extraction with ICSI for men with non-obstructive azoospermia has been performed in several centers over the last few years and has had a highly successful pregnancy rate. ${ }^{2}$ The success of this procedure has led to a reduced frequency of artificial insemination with donor sperm (AID). However, for couples seeking a healthy baby, AID remains an attractive alternative for several reasons, including a risk of Sertoli cellonly syndrome (there are no germ cells in testis), the high incidence of failed ICSI procedures and the increased genetic risks for offspring of ICSI fertilization.

The first human sperm bank in Mainland China was established in 1981 in Hunan Province and, currently, there are 11 sperm banks licensed by the Chinese government. All human sperm banks are state-owned and perform the following tasks: (i) organize and screen sperm donors, cryopreserve semen samples and provide these to qualified reproductive clinics; (ii) cryostore sperm to preserve male fertility for those who need it; ${ }^{3}$ and (iii) perform scientific research on reproductive health. A standard protocol for human sperm banking was initiated in 2001 by the Chinese Ministry of Health. Most guidelines published by the Chinese Ministry of Health have been either adapted or referenced from those of other countries. Some detailed procedures of China's sperm banking program differ greatly from those of Western countries; for example, sperm donation is completely anonymous, only donors between the ages of 22 and 44 years are eligible for selection, donor sperm cannot be provided to single women or same-sex couples, and each sperm donor can only impregnate up to 5 women via AID or in vitro fertilization. ${ }^{4}$

In April 2005, the Chinese Ministry of Health implemented a significant change to its policy based on UK legislation. The new policy

\footnotetext{
${ }^{1}$ Department of Urology, Renji Hospital, Shanghai Jiao Tong University School of Medicine, Shanghai Human Sperm Bank, Shanghai 200001 , China; ${ }^{2}$ Institute of Human Reproduction and Stem Cell Engineering, Human Sperm Bank, Central South University, Changsha 410078, China; ${ }^{3}$ Zhejiang Institute of Planned Parenthood Research and Zhejiang Human Sperm Bank, Hangzhou 310012, China and ${ }^{4}$ Human Sperm Bank, The Third Affiliated Hospital of Zhengzhou University, Zhengzhou 450052, China * The two authors contributed equally to this work. Correspondence: Dr Z Li (doc.zheng.li@gmail.com)

Received: 29 December 2010; Revised: 1 February 2011; Accepted: 22 February 2011; Published online: 30 May 2011
} 
requires that any donor of either gametes or embryos used in the treatment of other people agrees to disclose their identity to any offspring reaching 18 years of age. ${ }^{5}$ In the United States, a limit of 25 children per population of 800000 for a single donor is recommended by the American Society for Reproductive Medicine. ${ }^{6}$

Regulations and policies involving sperm donation differ from country to country due to cultural, social and economic factors. Since sperm donation is vastly influenced by sociocultural environment and its related policies, many concerns surrounding these issues are quite unique to China; these have not previously been well studied. Through investigation of the current state of semen donation in China and its clinical applications, we can obtain an overall understanding of China's sperm donation program and determine its limiting factors so that improvements and suggestions can be made to influence current policy.

\section{MATERIALS AND METHODS}

This study was approved by the Reproductive Ethics Committee of Renji Hospital, Shanghai Jiao Tong University School of Medicine. All donors signed informed consent during their first visit, agreeing that their semen samples or data could be used by the human sperm bank for scientific research. Four major human sperm banks (Shanghai Human Sperm Bank, Renji Hospital, Shanghai Jiao Tong University School of Medicine, Shanghai; Institute of Human Reproduction and Stem Cell Engineering and Human Sperm Bank, Central South University, Changsha; Zhejiang Institute of Planned Parenthood Research and Zhejiang Human Sperm Bank, Hangzhou; Human Sperm Bank, The Third Affiliated Hospital of Zhengzhou University, Zhengzhou) were chosen for this study. The study consisted of three parts: (i) data from all donors were reviewed and relevant demographic and clinical information was collected and analyzed. Demographic information included age, marital status, occupation, educational attainment, fertility history and reasons for withdrawal during the screening process. Clinical information included semen parameters pre- and post-thaw, artificial reproductive cycle numbers and treatment outcome; (2) a questionnaire was designed to investigate factors that influence donor attendance to the program, to assess knowledge about semen donation, and to assess attitudes and worries associated with donation. The questionnaire was distributed between January 2009 and June 2009 to a random sample of the initial donors and was completed anonymously and voluntarily at their first sperm bank visit just prior to being counseled on donor issues. All donors were also informed that responses to their questionnaire would not influence the sperm donation selection process; (iii) a follow-up of the clinical use of the donated semen and its therapeutic outcome was conducted.

\section{Criteria and screening of sperm donors in China}

The primary guidelines of anonymous sperm donor selection, published by the Chinese Ministry of Health in 2003 (http://www.moh.gov.cn/ publicfiles/business/htmlfiles/mohbgt/pw10303/200804/18593.htm), are the following: (i) donors must be between 22 and 44 years of age; (ii) donors must be in good health, based on both physical examination and psychological evaluation by qualified doctors, and have no history of genetic disease in their family; (iii) fresh semen is required to have a liquefaction time $<60 \mathrm{~min}$, sperm concentration $\geqslant 60 \times 10^{6}$ sperm $\mathrm{ml}^{-1}$, motility (percentage of rapid and slow progressive sperm) $\geqslant 60 \%$ and percentage of normal morphology $>30 \%$; (iv) post-thaw semen is required to have a motility $\geqslant 40 \%$, number of motile sperm per vial $\geqslant 12 \times 10^{6}$ and frozen-thaw survival rate $\geqslant 60 \%$; (v) potential donors must undergo laboratory testing to exclude individuals at high risk for sexually transmitted infections and genetic diseases, including; HIV-1 and -2, hepatitis B and C, syphilis, gonorrhoea, mycoplasma, chlamydia, cytomegalovirus, Toxoplasma gondii, rubella virus, herpes simplex virus types 1 and 2, and karyotype analysis. If the results from all tests are negative, the donation process begins and semen samples are cryopreserved. The samples must be cryopreserved for a minimum 6month quarantine period prior to allowing for HIV rescreening.

\section{Management of donors}

During the entire donation procedure (Figure 1), the health status of potential donors is carefully screened and monitored to reduce the risk of transmitting infectious agents to women during insemination. Since economic levels vary across regions of China, monetary compensation to donors for their time and expenses ranges from RMB3500 to RMB4000 (approximately US\$520-600) for the entire contracted donation period. The donors' confidentiality is assured and well protected. Only minimal information about the donor, including his age, appearance, education, profession, hobbies, blood type and $\mathrm{Rh}$ factor, is provided to the recipient.

\section{Records keeping}

In China, sperm banks are required to maintain permanent records about the initial selection process and subsequent tests and evaluations of each donor. Clinical outcomes (pregnant or not) for each treatment

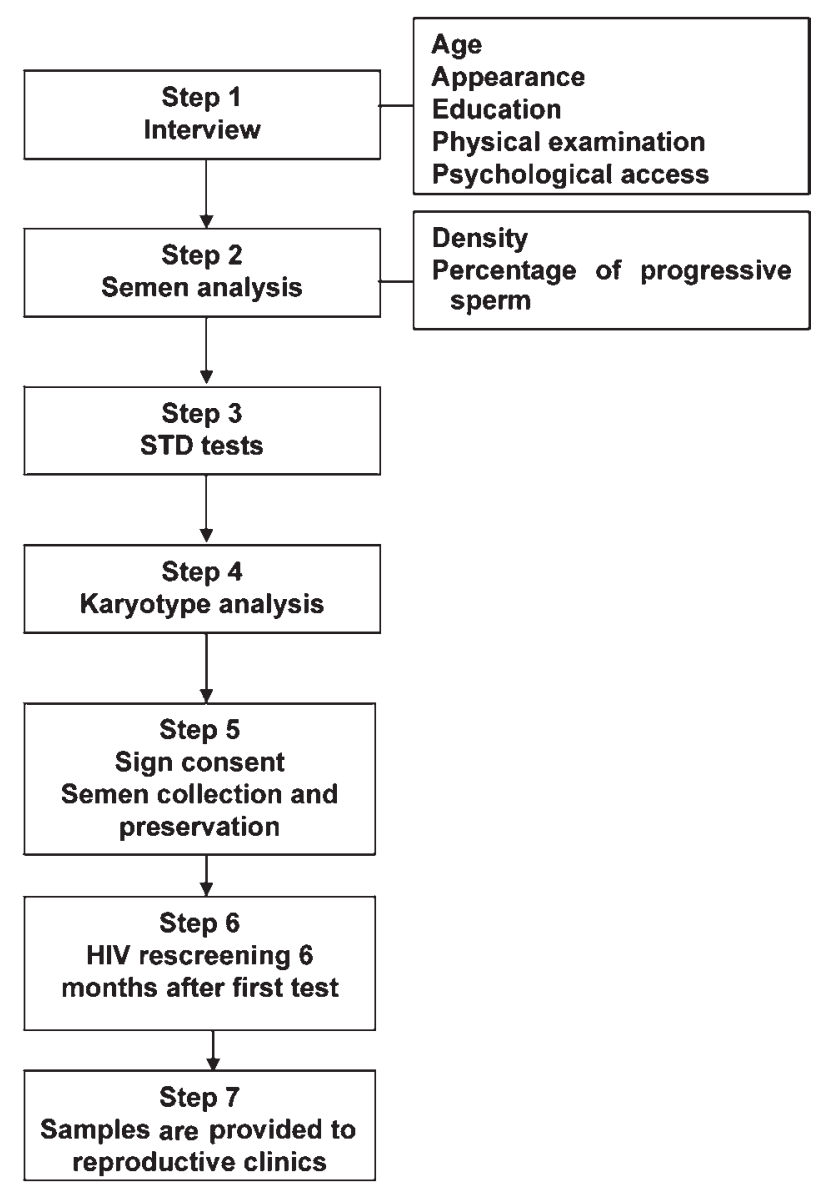

Figure 1 The complete procedure of donor screening and donation in human sperm banks in Mainland China. STD, sexually transmitted disease. 
cycle (AID and in vitro fertilization-ET) and outcome of fertility (abortion, healthy live birth or birth defect) must also be recorded.

\section{Data and questionnaire review}

Between January 2003 and December 2009, 19471 potential donors were contacted by the four major sperm banks participating in this study. Following the screening procedure, 33.2\% of donors (6467/ 19 471) were eligible for donation. General donor information, including age, marital status, occupation, educational attainment, fertility history, reasons for withdrawal during the screening process, semen parameters (pre- and post-thaw) and outcome of each artificial reproductive cycle, were reviewed and analyzed. A questionnaire aimed at investigating donor comprehension, intention and concerns about sperm donation was distributed to 516 potential donors and had a $100 \%$ response rate. Following their completion, anonymous returned questionnaires were collected for detailed analysis. The questionnaire was designed to seek information from donors regarding their knowledge of sperm donation and how they had heard about it, their motivation for sperm donation, and their concerns or worries about sperm donation that influenced their decision to donate. The questionnaire was semistructured and questions were primarily in multiple-choice format.

\section{RESULTS}

\section{Donor}

Between January 2003 and December 2009, there were a total of 19471 male volunteers of $22-40$ years of age, with a mean age of $25.7 \pm 8.5$ years, seeking to become semen providers at the four human sperm banks participating in this study. Approximately 87.6\% (17 062/ 19471 ) of these candidates were between 22 and 25 years of age, $9.3 \%(1818 / 19471)$ were between 26 and 30 years of age, $2.7 \%$ (517/19 471) were between 31 and 35 years old, and $0.4 \%$ (74/ 19471 ) were between 36 and 40 years old. The majority of candidate donors (95.2\%, (18 536/19 471)) were not married and 99.1\% (19 296/ 19 471) did not have children. College students made up the largest occupational category among the candidates (92.7\%, (18 049/ $19471)$ ), while only a small percentage were office staff or trades and medical professionals. After strict screening, 33.2\% (6 467/ 19 471) of potential donors were qualified and recruited to the program. The main reasons for non-recruitment were unacceptable semen parameters $(55.0 \%,(10709 / 19471))$ or a positive sexually transmitted disease testing result (7.9\%, (1538/19 471)). Around $48.1 \%$ (9375/19 471) of donors did not meet the criteria for fresh semen specimens, while 6.9\% (1345/19 471) failed the post-thaw semen analysis. Among those disqualified because of a positive STD test, hepatitis B virus, mycoplasma, cytomegalovirus and chlamydia were the most common infections (Table 1). There were 327 qualified

Table 1 Distribution and percentage of each STD category among 1530 donors not recruited due to STDs

\begin{tabular}{lc}
\hline Category & No. (\%) \\
\hline HBV & $670(43.8)$ \\
Mycoplasma & $496(32.4)$ \\
Cytomegalovirus & $171(11.2)$ \\
Chlamydia & $162(10.6)$ \\
Syphilis & $14(0.9)$ \\
HCV & $8(0.5)$ \\
Gonorrhoea & $6(0.4)$ \\
HIV & $3(0.2)$ \\
\hline
\end{tabular}

Abbreviations: HBV, hepatitis B virus; HCV, hepatitis C virus; STD, sexually donors who withdrew from the program. The primary reasons for withdrawal were frustration due to repeated results that did not achieve the required pre- or post-thaw semen parameters, participation in the donor program merely to receive free physical examination and medical tests (these candidates ceased providing sperm after receiving their test results), and job transfers to other cities.

There were a total of 516 initial donors who participated in the survey. All questionnaires were completed anonymously and collected for further analysis. All donors were motivated to participate in sperm donation programs by a desire to assist infertile couples (100\%, 516/ $516)$, and a majority $(90.9 \%, 469 / 516)$ also mentioned monetary compensation as a second influence that contributed to their participation. Regarding donor initial awareness and understanding of semen donation, $64.9 \%(335 / 516)$ first heard about the sperm donation program through personal contact with staff, former donors or printed advertisements distributed by the sperm bank. An additional 27.9\% (144/516) of donors received recruitment information from sperm bank websites and through the media. Newspaper articles were also a common source of information for donors. Approximately $93.4 \%$ (482/516) of donors showed some concerns or worries about sperm donation. The most common concern was the risk of inadvertent halfsibling unions between offspring of the same anonymous donor. Another common question was whether the donors themselves, or the recipients and their offspring, could access information about each other (Table 2). After explanation by sperm bank staff members, most donors had a better understanding of this policy and their worries were quelled.

\section{Clinical outcomes of donation}

Between January 2003 and December 2009, a total of 488389 vials of donor sperm were cryopreserved in the four sperm banks participating in this study. The average sperm concentration of fresh samples was $(78.7 \pm 25.3) \times 10^{6}$ sperm $\mathrm{ml}^{-1}$, and the average motility rate was $62.1 \% \pm 15.3 \%$. Post-thaw specimens had an average sperm concentration of $(58.2 \pm 26.3) \times 10^{6}$ sperm $\mathrm{ml}^{-1}$, an average motility rate of $42.6 \% \pm 13.5 \%$, and an average frozen-thawed survival rate (pre-thaw motility/post-thaw motility) of $72.8 \% \pm 19.7 \%$. The four sperm banks provide semen specimens to 21 reproductive centers. Among 36438 AID cycles, there were 8712 (23.9\%) clinical pregnancies, 1063 (2.9\%) abortions, 6048 (16.6\%) live births and $45(0.1 \%)$ congenitally malformed foetuses. Among 7148 in vitro fertilization cycles, there were $3273(45.8 \%)$ clinical pregnancies, 361 (5.1\%) abortions, 2519 (35.2\%) live births and $23(0.3 \%)$ congenitally malformed foetuses.

\section{DISCUSSION}

Infertility is an important health problem, and the demand for assisted reproductive technology has increased rapidly over the past several decades. Human sperm donation and cryopreservation can serve as a remedy for the lack of male gametes in infertile couples and satisfy the male partner's desire to have a child despite the absence of a biological, or genetic, link. The population of China is 1324655000 and, according to the reported incidence of infertility, nearly $10 \%$ are infertile. The majority of infertile couples have to rely on assisted reproductive

Table 2 Donors' concerns about sperm donation distribution ( $n=516$ )

\begin{tabular}{lc}
\hline Concern raised & Frequency, $\mathrm{n}(\%)$ \\
\hline Unwitting sibling mate & $461(89.3)$ \\
Disclosure of donor information & $372(72.1)$ \\
Others & $53(10.2)$ \\
\hline
\end{tabular}


technology to have offspring, and a small proportion with severe male infertility turn to AID. Over the past 30 years, the number of human sperm banks has increased to 11 units; this growth and development has had a significant impact on reproductive medicine in China, a field that has also progressed rapidly over this time period. In Mainland China, sperm banks are closely monitored and carefully administrated by the central and local health ministry units. All sperm banks are inspected and assessed every 2 years. If mandatory standards are not met, recruitment and donations are suspended until all requirements are met.

In large cities, including Beijing, Shanghai and Guangzhou, more than 10000 couples hope to undergo AID. There are now approximately 30 reproductive centers in Mainland China that are qualified to perform AID treatments. The average wait time for therapeutic donor insemination at these centers is 1.5-2.0 years, a wait time that has caused some couples to abandon their plans for AID. Although both the scale of semen storage and the quantity of donations have continued to increase over the past several years, there is still a striking gap between donor supply and recipient demand. This imbalance can be attributed to several factors.

First, among these factors is the stringent screening procedure for STD and the high semen quality standards. The screening criteria for semen parameters are much higher (threefold) than those recommended in the WHO laboratory manual (4th edition). ${ }^{7}$ Because of this high threshold, $48.1 \%$ of candidate sperm donors are disqualified from participation in sperm donation programs. STDs are the second most common reason for donation failure. In China, there are estimated to be approximately 120000000 hepatitis B virus carriers, and many donor candidates fail because they test positive for hepatitis $B$ surface antigens. Other common STDs among candidate donors are mycoplasma and chlamydia. Because social attitudes towards premarital sexual behavior have become much more tolerant, the risk of STDs, particularly mycoplasma and chlamydia, has increased among unmarried college students. While some donors returned to donation centers with negative results after a period of treatment, others were rejected because of repeated positive results.

Second, social and cultural factors in China have contributed to the large gap between sperm supply and recipient demand. Although all donors agreed that sperm donation is not harmful and that it is a humanitarian contribution that will help infertile couples, because they are passing on their genetic material to a new life, they do not consider semen donation to be analogous to blood donation. In China, the notion of sperm donors serving as the genetic link between a husband and his family is often perceived to be extremely embarrassing. ${ }^{8}$ On the other hand, especially in rural areas in China, infertility is considered shameful for the entire family, causing infertile couples to suffer greatly from additional social pressures. Babies born to infertile couples through therapeutic donor insemination not only help to salvage a couple's happiness but are also important to the entire family. For these reasons, sperm banks should emphasize the humanitarian contributions provided by donors to assist them in overcoming their ethical apprehensions.

Third, the policy limiting the number of children that can be born from one donor has added to the gap between donor supply and recipient demand. The majority of countries that support the use of donor insemination in assisted reproductive technology set donor limits based on the number of offspring born from one donor, so that the inadvertent risk of half-sibling unions between the offspring of anonymous donors can be controlled. These limits vary considerably from country to country, and there is a lack of data to support them. ${ }^{9}$ Considerations in establishing these limits include the size of the country's population and its density and mobility. Published mathematical models used to determine sperm donor limits are outdated and inadequate for present-day use. A more appropriate, internationally recognized model is needed to accurately set acceptable limits. ${ }^{10}$ The American Society for Reproductive Medicine recommends a limit of 25 children per population of 800000 for each donor. ${ }^{11}$ In the United Kingdom, current guidelines specify that sperm from a single donor should not be used to produce children for more than 10 families. ${ }^{12}$ Compared to the policies of Western countries and considering China's population density and size, the five-pregnancy limit from one donor's sperm requires re-evaluation to achieve a both cost-effective and safe policy.

Anonymous donor sperm banking has been a fundamental aspect of reproductive medicine for several decades; in recent years, there has been a growing trend encouraging both openness and disclosure of donor identification to donor recipients to benefit their offspring. ${ }^{13}$ In Western Australia, the total number of sperm donors was reduced by $50 \%$ and the rate of new donors declined $32 \%$ following the passage of a law requiring the release of identifying donor information to mature offspring in December 2004. ${ }^{14}$ Since April 2005, gamete donors in the United Kingdom have been required to be willing to be identified to their offspring should the offspring request this information in the future. This change in legislation has led to considerable anxiety about the future availability of potential donors. ${ }^{15}$ In The Netherlands, a similar decline has also been reported. However, while the number of infertile couples requesting AID has remained constant, the gap between those providing donations and those requesting AID has widened. ${ }^{16,17}$ In the United States, a 'double track' policy has become increasingly popular. Under this policy, the donor can choose to be either an anonymous or an identified donor. ${ }^{11}$ In Mainland China, only an anonymous donor system is currently in place because of traditional cultural and philosophical views regarding the genetic or biological origin of the child. The subject of donor identification remains a sensitive issue because it challenges the biological/genetic affiliation of the family. Recipient couples prefer to keep the identity of sperm donors from offspring as well as from other people. Similarly, donors themselves also prefer to maintain their privacy and do not want any contact (emotional, financial or legal) with their offspring. The anonymous donation policy in Mainland China will likely continue because of traditional Chinese family values and beliefs, which are quite different from those of Western society. If the openidentification policy were to be implemented in China, the shortage of sperm donors would likely become even more prominent.

Commercialization of human gametes is now legally prohibited in China and all sperm banks are non-profit organizations and centers. Donors must be altruistically motivated to donate gametes, and should not sell their sperm simply as if it were a commodity. China is neither the first nor the only country in the world to have legislation that supports voluntary gamete donation. Other countries have adopted similar systems through either legislative changes or the implementation of best-practice models. In 2004, Canada's Assisted Human Reproduction Act was changed to make it illegal for sperm banks to pay for sperm. ${ }^{18}$ Following its enactment, the number of sperm donors declined, ${ }^{19}$ and the number of sperm banks decreased from 40 to only a single center, the Toronto Institute for Reproductive Medicine, located in Ontario. Approximately $80 \%$ of Canada's donor sperm is imported from the United States, where a higher level of monetary compensation is allowed for participating sperm donors. In 2006, the United Kingdom banned payment for sperm donors, a policy that caused the sperm donor pool to shrink dramatically. In the United States, there are three types of anonymous donor sperm banks: 
(i) physician practice-based; (ii) hospital/clinic-based; and (ii) commercial corporations, which are the largest and the for-profit banks. ${ }^{20}$ A study to examine factors associated with sperm donor recruitment and motivation found that all sperm donation providers shared the desire to assist infertile couples. ${ }^{21}$ While this was their primary motivating factor, at least $50 \%$ of these donors felt that they should receive payment for their donation and noted that reimbursement for their expenses was an important determinate of their participation. In the present study, we found that the majority of donors donated sperm because they wanted to help others. The procedure of sperm donation is quite different from blood and bone marrow donation. Sperm donors must sacrifice both a significant amount of time (usually several months) and energy to complete this procedure. Because of these demands, they deserve reasonable reimbursement for the expenses incurred during their visits to the sperm bank. In Mainland China, sperm banks are usually established in the nation's larger cities. As university towns are often located in rural areas that are far from downtown, it can be quite inconvenient for donors to visit donation centers. Reasonable payment for their participation in sperm donation program would compensate donors for both their time and travel fees and also serve as encouragement and incentive for their participation.

\section{CONCLUSION}

The results from the present study provide insight to the current status of sperm donation and its clinical use in Mainland China. These results show that, relative to policies in place in other nations, China's sperm banks and the clinical use of donor sperm specimens are strictly administrated, and sperm donation has now reached a stable and disciplined stage. The results also revealed that there is a significant gap between the supply and demand of semen specimens due to China's stringent screening criteria, social and culture pressures, and sperm donor limits. Public promotion and enlightenment through mass media to correct false notions and modify policies concerning donor limits will enhance future recruitment of potential donor and help to close the gap between those providing and requesting sperm donations. Because of China's unique cultural and social traditions, the anonymous donation system should continue, and reasonable monetary compensation is necessary to encourage the interests of both the donor and the recipient.

\section{AUTHOR CONTRIBUTIONS}

ZL jointly conceived the study with PP and WBZ, and supervised the project with YRH. PP and WBZ implemented the study and prepared the manuscript, and XZZ, YSL, QXW, XRC, YL and HLD conducted data analysis. All authors contributed extensively to the work presented in this paper.

\section{COMPETING FINANCIAL INTERESTS}

The authors declare no competing financial interests.

\section{ACKNOWLEDGMENTS}

The research was supported by the Shanghai Scientific Committee Program (No. 08DZ2292901), the Shanghai Jiao Tong University Med-Science Cross Research Foundation (No. YG2010MS88) and the Science and Technology Commission of Shanghai Municipality Program (Nos. 08410701700 and 09411950300).

1 Anger JT, Gilbert BR, Goldstein M. Cryopreservation of sperm: indication, methods and results. J Urol 2003; 170: 1079-84.

2 Aydos K, Demirel LC, Baltaci V, Unlu C. Enzymatic digestion plus mechanical searching improves testicular sperm retrieval in non-obstructive azoospermia cases. Eur J Obstet Gynecol Reprod Biol 2005; 120: 80-6.

3 Ping $\mathrm{P}$, Zhu WB, Zhang XZ, Yao KS, Xu $\mathrm{P}$ et al. Sperm banking for male reproductive preservation: 6 years' retrospective multicenter study in China. Asian J Androl 2010; 12: 356-62.

4 GonGong D, Liu YL, Zheng Z, Tian YF, Li Z. An overview on ethical issues about sperm donation. Asian J Androl 2009; 11: 645-52.

5 Mahlstedt PP, Labounty K, Kennedy WT. The views of adult offspring of sperm donation: essential feedback for the development of ethical guidelines within the practice of assisted reproductive technology in the United States. Fertil Steril 2010; 93: 2236-46.

6 Critser JK. Current status for semen banking in the USA. Hum Reprod 1998; 13: 5567.

7 WHO. WHO Laboratory Manual for the Examination and Processing of Human Semen. 4th ed. New York: Cambridge University Press; 1999.

8 Liao J, Dessein B, Pennings G. The ethical debate on donor insemination in China. Reprod Biomed Online 2010; 20: 895-902.

9 Sawyer N. Sperm donor limits that control for the 'relative' risk associated with the use of open-identity donors. Hum Reprod 2010; 25: 1089-96.

10 Sawyer N, McDonald J. A review of mathematical models used to determine sperm donor limits for infertility treatment. Fertil Steril 2008; 90: 265-71.

11 The American Society for Reproductive Medicine. Guidelines for sperm donation. Fertil Steril 2004; 82: S9-12.

12 Human Fertilisation and Embryology Authority. Who are the Donors? An HFEA Analysis of Donor Registrations and Use of Donor Gametes over the Last 10 Years. London: Human Fertilisation and Embryology Authority; 2005.

13 Burr JA. To name or not to name? An overview of the social and ethical issues raised by removing anonymity from sperm donors. Asian J Androl 2010; 12: 801-6.

14 Godman KM, Sanders K, Rosenberg M, Burton P. Potential sperm donors', recipients' and their partners' opinions towards the release of identifying information in Western Australia. Hum Reprod 2006; 21: 3022-6.

15 Daniels K. Anonymity and openness and the recruitment of gamete donors. Part I: Semen donors. Hum Fertil (Camb) 2007; 10: 151-8.

16 Janssens PM, Simons AH, van Kooij RJ, Blokzijl E, Dunselman GA. A new Dutch Law regulating provision of identifying information of donors to offspring: background, content and impact. Hum Reprod 2006; 21: 852-6.

17 Paul S, Harbottle S, Stewart JA. Recruitment of sperm donors: the Newcastle-uponTyne experience 1994-2003. Hum Reprod 2006; 21: 150-8.

18 Daniels K, Feyles V, Nisker J, Perez-y-Perez M, Newton C et al. Sperm donation: implications of Canada's Assisted Human Reproduction Act 2004 for recipients, donors, health professionals, and institutions. J Obstet Gynaecol Can 2006; 28: 608-15.

19 del Valle AP, Bradley L, Said T. Anonymous semen donor recruitment without reimbursement in Canada. Reprod Biomed Online 2008; 17: 15-20.

20 Critser JK. Current status of semen banking in the USA. Hum Reprod 1998; 13: 5567.

21 Lalos A, Daniels K, Gottlieb C, Lalos O. Recruitment and motivation of semen providers in Sweden. Hum Reprod 2003; 18: 212-6. 